\title{
Method of Using Dual-Booting and Multi-Booting of Microsoft Windows Family Operating Systems on External System Drive
}

\author{
Vasyl Kostogriz \\ Taras Shevchenko National University of Kyiv, \\ Faculty of Computer Science and Cybernetics, \\ Department of Information Systems, \\ 03680, Ukraine, Kyiv, 4d Academician Glushkov avenue, \\ bioinfo@univ.net.ua
}

\begin{abstract}
Computer's operating system in generally installed on its internal SSD (HDD). When user boot computer, the BIOS loads the boot loader from the internal SSD and the boot loader boots the installed operating system. A boot loader is a computer program that loads an operating system or some other system software for the computer after completion of the power-on selftests. A boot loader is loaded into main memory from persistent memory. On modern general purpose computers, the boot up process can take tens of seconds, or even minutes, and typically involves performing a power-on self-test, locating and initializing peripheral devices, and then finding, loading and starting an operating system. Booting is complete when the normal, operative, runtime environment is attained.

Dual-booting is a technique which allows a single physical computer to run two operating systems.

Multi-booting allows to the common configuration of multiple operating systems on computer, and ability to choose which operating system to boot.

I have Implemented method of using dual-booting and multibooting based on the external SSD system drive.
\end{abstract}

Index Terms - System drive; HDD; SSD; operating system; installation files; WIM-file; DISM; Diskpart; BCDboot; Microsoft Windows.

\section{INTRODUCTION}

An external drive is just a hard drive (HDD) or solid-state drive (SSD) that is connected to a computer on the outside rather than on the inside.

Some external drives draw power over their data cable, which of course comes from the computer itself, while others may require an $\mathrm{AC}$ wall connection to derive power on their own.

One way to think of an external hard drive is as if it were a regular, internal hard drive that has been removed, covered in its own protective casing, and plugged into the outside of your computer.

Internal hard drives can even be converted into external hard drives via what's called a hard drive enclosure.

External hard drives come in varying storage capacities, but they all connect to a computer either by USB, FireWire, eSATA, or wirelessly.
External hard drives are sometimes called portable hard drives. A flash drive is one common, and very portable, type of external hard drive.

External hard drives are portable, easy to use, and can provide a large amount of storage whenever you need it. You can store the actual device any place you like, and carry a large number of files with you wherever you go.

Another advantage of owning an external drive is that you can move them from computer to computer, making them great for sharing large files.

Because of their usually large storage capacities (often in the terabytes), external hard drives are often used to store backed up files.

It's common to use a backup program to back up things like a music, video, or picture collection to an external drive for safe keeping, separate from the originals in case they're accidentally changed or deleted.

Even if not used for backup purposes, external hard drives provide an easy way to expand your existing storage without having to open up your computer, which is especially difficult if using a laptop.

External hard drive can also be used to provide additional storage to an entire network (though internal hard drives are usually more common in these scenarios). These kinds of network storage devices can be accessed by numerous users at once and often serve as a way for users to share files within a network to avoid emailing or uploading the data online.

Internal hard drives are connected directly to the motherboard, whereas external storage devices first run through the outside of the computer case, and then directly to the motherboard.

Operating systems and software installation files are generally installed to internal drives, while external hard drives are used for non-system files, like photos, videos, documents, and files of those types.

Internal hard drives draw power from the power supply inside a computer. External hard drives are powered either through their data cable or via dedicated AC power.

Data can be compromised much easier if it's stored on an external hard drive because they're generally located on a desk 
or table, making them very easy to pick up and steal. This is different than an internal hard drive where the entire computer has to be taken, or the hard drive removed from the inside, before someone can have physical access to your files.

External hard drives are also generally moved around more than internal ones, causing them to fail more easily due to mechanical damage. SSD based drives, like flash drives, are less prone to this sort of damage.

SSDs are dramatically coming into our computing life and more and more users are benefit from the new technology. SSDs tend to be with large capacity and affordable prices on the market. SSD manufacturers like Samsung, Intel, Toshiba, Kingston, SanDisk, and PNY are well-known SSD brands. For a period of time, replace HDD with SSD in desktop and laptop becomes a popular trend. This part of PC users mostly want to change old and slow traditional hard drive with new and fast solid state drive. Of course, some of them use SSD for OS and HDD for storage to fully take advantage of each disk.

SSD technology primarily uses electronic interfaces compatible with traditional block input/output (I/O) hard disk drives (HDDs), which permit simple replacements in common applications. New I/O interfaces like SATA Express and M.2 have been designed to address specific requirements of the SSD technology.

SSDs have no moving mechanical components. This distinguishes them from traditional electromechanical drives such as hard disk drives (HDDs) or floppy disks, which contain spinning disks and movable read/write heads. Compared with electromechanical drives, SSDs are typically more resistant to physical shock, run silently, have quicker access time and lower latency [1].

\section{DUAL-BOOTING AND MULTI-BOOTING}

The term dual-booting refers to the common configuration of specifically two operating systems. Multi-booting is the act of installing multiple operating systems on a computer, and being able to choose which one to boot. Multi-booting may require a custom boot loader.

Multi-booting allows more than one operating system to reside on one computer, for example if you have a primary operating system and an alternate system that you use less frequently. Another reason for multi-booting can be to investigate or test a new operating system without switching completely. Multi-booting allows a new operating system to configure all applications needed, and migrate data before removing the old operating system, if desired. A possible alternative to multi-booting is virtualization, where a hypervisor is used to host one or more virtual machines running guest operating systems. Multi-booting is also useful in situations where different software applications require different operating systems. A multi-boot configuration allows a user to use all of this software on one computer. This is often accomplished by using a boot loader such as NTLDR, LILO, or GRUB which can boot more than one operating system. Multibooting is also used by software developers when multiple operating systems are required for development or testing purposes. Having these systems on one machine is a way to reduce hardware costs.
In a multi-boot computer each of the multiple operating systems can reside on its own storage device, or some storage devices might contain more than one operating system in different partitions.

An example of a computer with one operating system per storage device is a dual-booting computer that stores Windows on one disk drive and Linux on another disk drive. In this case a multi-booting boot loader is not strictly necessary because the user can choose to enter BIOS configuration immediately after power-up and make the desired drive first in the boot-order list. However, it is more convenient to have a multi-booting boot loader on one of the drives, set BIOS once to always start booting from (i.e., load the boot loader from) that drive, and then allow the user to choose an operating system from that boot loader's menu. No special disk partitioning is necessary when each operating system has its own dedicated disk drive.

An example of a computer with multiple operating systems per storage device is a dual-booting computer that stores both Windows and Linux on the same disk drive. In this case a multi-booting boot loader is necessary. Also, the disk must be partitioned to give each operating system its own partition on the disk drive.

The basic concept involves partitioning a disk to accommodate each planned installation, usually including separate partitions for boot, root, data storage and backups [2].

\section{DISKPART. DISM. BCDBOOT}

DiskPart is a text-mode command interpreter in Windows OS that enables you to manage objects, such as disks, partitions, or volumes by using scripts or direct input at a command prompt.

DiskPart can be found under the \%WINDIR\%\System $32 \backslash$ folder. DiskPart is also included in a typical Windows Preinstallation Environment (Windows PE).

Before you can use DiskPart commands on a disk, partition, or volume, you must first list and then select the object to give it focus. When an object has the focus, any DiskPart commands that you type act on that object.

You can list the available objects and determine the number or drive letter of an object by using the list disk, list volume, and list partition commands. The list disk and list volume commands display all disks and volumes on the computer. However, the list partition command displays only partitions on the disk that have the focus. When you use the list commands, an asterisk $\left(^{*}\right)$ appears next to the object that has the focus. You select an object by its number or drive letter, such as disk 0 , partition 1 , volume 3 , or volume $\mathrm{C}$.

When you select an object, the focus remains on that object until you select a different object. Some commands automatically change the focus.

You can give focus only to a partition on the selected disk. When a partition has the focus, the related volume (if any) also has the focus. When a volume has the focus, the related disk and partition also have the focus if the volume maps to a single specific partition. If this is not the case, the focus on the disk and partition is lost.

When using the DiskPart command as a part of a script, is recommended to complete all the DiskPart operations together as part of a single DiskPart script. You can run consecutive 
DiskPart scripts. However, you must allow for at least 15 seconds between each script for a complete shutdown of the previous execution before you run the DiskPart command again in successive scripts. Otherwise, the successive scripts might fail. You can add a pause between consecutive DiskPart scripts by adding the time-out $/ \mathrm{t} 15$ command to your batch file together with your DiskPart scripts [3].

The Deployment Image Servicing and Management (DISM) platform is used to mount and service Windows images before deployment. A subset of DISM commands can be used on online Windows images. You can use DISM tools to mount, and get information about, Windows image (.wim) files or virtual hard disks (.vhd or .vhdx). You can also use it to install, uninstall, configure, and update Windows features, packages, and drivers in a Windows image or to change the edition of a Windows image.

The DISM platform also includes a command-line tool, DISM.exe, and the DISM API [4].

The DISM commands can be used to mount, unmount, capture, append, and delete and query .wim, .vhd and .vhdx files. These options are not case sensitive.

For WIM, the DISM / Apply-Image command applies a Windows image file (.wim) or a split Windows image (.swm) files to a specified partition. Beginning with Windows 10 , version 1607 , DISM can apply and capture extended attributes (EA).

This option doesn't support applying an image from a virtual hard disk (VHD), though you can use this command to apply images to a .vhdx file that's been attached, partitioned, and formatted.

Arguments for WIM:

DISM.exe

/Apply-Image

/ImageFile: $<$ path_to_image_file $>$ $[/$ SWMFile: $<$ pattern $>$ ] /ApplyDir: $<$ target_directory $>\quad\{/$ Index: $<$ image_index $>$ | /Name: $<$ image_name $>\} \quad$ [/CheckIntegrity] [/Verify] [/NoRpFix] [/ConfirmTrustedFile] [/WIMBoot (deprecated)] [/Compact] [/EA]

Examples:

Dism /apply-image /imagefile:install.wim /index:1 /ApplyDir:D:।

Dism /apply-image /imagefile:install.swm /swmfile:install.swm /index:1 /applydir:D: [5]

The BCDboot tool is a command-line tool that enables you to manage system partition files. You can use the tool in the following scenarios:

- $\quad$ Setting up a system partition when you deploy new computers. For more information, see Capture and Apply Windows Images.

- $\quad$ Setting up Windows ${ }^{\circledR}$ to boot to a virtual hard disk. For instructions, see Walkthrough: Deploy a Virtual Hard Disk for Native Boot.

- Repairing the boot environment located on the system partition. If the system partition has been corrupted, you can use BCDboot to replace the system partition files with new copies of these files from the Windows partition.
To configure the system partition, BCDboot copies a small set of boot-environment files from the installed Windows image to the system partition. Next, BCDboot creates a Boot Configuration Data (BCD) store on the system partition that instructs the computer to boot to the Windows partition.

BCDboot uses the \%WINDIR\% $\$ System $32 \backslash$ Config $\backslash B C D$ Template file to create a new BCD store and initialize the $\mathrm{BCD}$ boot-environment files on the system partition. You can define specific BCD settings in the BCD-Template file. The $\mathrm{BCDboot}$ tool also copies the most recent versions of bootenvironment files from the operating-system image $\%$ WINDIR\% $\%$ boot folder to the system partition.

BCDboot can update an existing boot environment on the system partition:

- BCDboot copies newer versions of files from the Windows image to the system partition.

- If a BCD store already exists on the system partition, BCDboot creates a new boot entry in the existing BCD store based on settings in the BCD-Template file.

- If there is already a boot entry for this Windows partition, by default, BCDboot erases the old boot entry and its values. To retain the values from an existing boot entry when you update the system files, you can use the $/ \mathrm{m}$ option together with the BDCBoot.exe command.

The following command-line options are available for BCDboot.exe.

BCDBOOT < source $>[/ 1<$ locale $>][/$ s < volume-letter $>$ [/v] [/m [\{OS Loader GUID $\}]]$

\section{THE NEW METHOD OF USING DUAL-BOOTING AND MULTI- BOOTING ON EXTERNAL SYSTEM DRIVE}

The method of using dual-booting and multi-booting based on external system drive consist of a few steps:

a) Prepare USB external SSD based on NTFS file system using diskpart tool:

- Insert a USB external SSD into a running computer.

- Open a Command Prompt window as an administrator.

- Type diskpart.

- In the new command line window that opens, to determine the USB external SSD number or drive letter, at the command prompt, type list disk, and then click ENTER. The list disk command displays all the disks on the computer. Note the drive number or drive letter of the USB external SSD.

- At the command prompt, type select disk $<\mathrm{X}>$, where $\mathrm{X}$ is the drive number or drive letter of the USB flash drive, and then click ENTER.

- Type clean, and the click ENTER. This command deletes all data from the USB flash drive.

- To create a new primary partition on the USB external $\mathrm{SSD}$, type create partition primary, and then click ENTER.

- To select the partition that you just created, type select partition 1, and then click ENTER. 
- Type active, and then click ENTER.

- To format the partition, type format $\mathrm{fs}=\mathrm{ntfs}$ quick, and then click ENTER.

- To assign a drive letter, type assign, and then click ENTER.

- Type exit, and then click ENTER.

b) Install Microsoft Windows 10 Enterprise 2016 LTSB on the USB external SSD (consist of external enclosure AGESTAR 31UB2A18 and SSD INTEL SS DSC2BB240G7):

1. Use the DISM tool to apply images to your Windows partition, run the DISM /apply-image /imageFile: $<$ image file $>$ /index:<index_number $>/$ ApplyDir: $<$ image_path $>$ command.

Example:

dism /apply-image /imagefile:d:|sourceslinstall.wim /index:1 /applydir:e:

2. Set up a basic system partition using the BCDboot tool to initialize boot configuration data that is used to start Windows.

USB external SSD system drive boot configuration based on next example:

\section{bcdboot C: \Windows /s S: /f ALL}

This example is taken from Microsoft site, it copies BCD files that support booting on either a UEFI-based or a BIOS-based computer from the C:IWindows folder to a USB flash drive that was assigned the volume letter $\mathrm{S}$ [7].

c) Create 3 VHDXs (intel.vhdx, intel2.vhdx, intel3.vhdx) on external SSD system drive from diskpart using next example (taken from Microsoft site) [8]:

1. From the Command Prompt, open Diskpart.

- Type diskpart, and then click ENTER.

2. Create and prepare a new VHDX.

- Type create vdisk file=C: $/$ windows.vhdx maximum $=25600$ type $=$ fixed, and then click ENTER.

3. Attach the VHDX. This adds the VHDX as a disk to the storage controller on the host.

- Type attach vdisk, and then click ENTER.

4. Create a partition for the Windows files, format it, and assign it a drive letter. This drive letter will appear in File Explorer.

- Type create partition primary

format quick label=vhdx

assign letter $=\mathrm{v}$, and then click ENTER.

\section{Exit Diskpart}

- Type exit, and then click ENTER.

d) Create 3 bootable USB flash drives with Microsoft Windows 8.1 Enterprise, Microsoft Windows Server 2012 R2, Microsoft Windows Server 2016. e) Install operating systems from bootable USB flash drives to 3 VHDXs (intel.vhdx, intel2.vhdx, intel3.vhdx) using MS Windows OS traditional installation process via attaching vhdx files as virtual disks to windows OS setup menu.

For attaching vhdx files need to use DiskPart tool via running cmd.exe from Windows OS setup menu.

After successfully complete of MS Windows OS installation process on the first VHDX file will be possible OS dual-booting, after complete of OS installation process on the second and third VHDX files - multi-booting will be present in OS boot menu for USB external SSD system drive.

The installation of Microsoft Windows 10 Enterprise LTSB was successfully applied to SSD INTEL SS DSC2BB240G7 based on external enclosure AGESTAR 31UB2A18. That gives a possibility to create an external system drive as a part of system architecture. An important possibility is applying of MS Windows OS traditional installation process to VHDX files that are located on the external system drive via attaching them to windows OS setup menu. Dual-booting and multi-booting on external system drive are results of the alternate applying of Microsoft Windows operating systems to external SSD as system drive and to VHDX files as virtual system drives.

For configuration of external SSD as a system drive were applied unpacked files of Windows 10 WIM-file and tools such as DISM, Diskpart, BCDboot.

BCDboot creates a Boot Configuration Data (BCD) store on the system partition that instructs the computer to boot to the Windows partition.

And for configuration dual-booting and multi-booting were applied VHD technology and operating systems, such as Windows 8.1, Windows Server 2012 R2, Windows Server 2016 on single partition of external SSD system drive. It has been found that the use of external SSD by this method, improves modernization of traditional computer architecture and new system functionality for external SSD.

\section{REFERENCES}

[1] Solid-state drive, 2018 Available at: https://en.wikipedia.org/wiki/Solid-state_drive.

[2] Multi-booting, 2018, Available at: https://en.wikipedia.org/wiki/Multibooting.

[3] DiskPart Command-Line Options (Standard 7 SP1), 2014, Available at: https://msdn.microsoft.com/enus/library/ff794606(v=winembedded.60).aspx.

[4] DISM, 2018, Available at: https://docs.microsoft.com/enus/powershell/module/dism/?view=win10-ps.

[5] DISM Image Management Command-Line Options, 2017, Available at: https://docs.microsoft.com/en-us/windows-

hardware/manufacture/desktop/dism-image-management-commandline-options-s14\#apply-image.

[6] BCDboot Command-Line Options, 2012, Available at: https://docs.microsoft.com/en-us/previous-versions/windows/itpro/windows- $7 / \mathrm{dd} 744347(\mathrm{v}=\mathrm{ws} .10)$.

[7] BCDBoot Command-Line Options, 2017, Available at: https://docs.microsoft.com/en-us/windowshardware/manufacture/desktop/bcdboot-command-line-options-techrefdi.

[8] Boot to a virtual hard disk: Add a VHDX or VHD to the boot menu, 2018, Available at: https://docs.microsoft.com/en-us/windowshardware/manufacture/desktop/boot-to-vhd--native-boot--add-a-virtualhard-disk-to-the-boot-menu. 